\title{
Public Health and Health System Reforms in Pakistan; An Ethical Perspective
}

\author{
Subhana Akber* \\ Health Services Academy, National Institute of Health (NIH), Islamabad, Pakistan \\ *Corresponding Author: Subhana Akber, Health Services Academy, National Institute of Health (NIH), Islamabad, Pakistan.
}

Received: September 26, 2019; Published: October 11, 2019

DOI: $10.31080 /$ ASMS.2019.03.0437

The health care system of any country plays a major role in developing a successful nation by maintaining and restoring health of its population at an individual level. Pakistan being the sixth most populous country of the world ranking at 147 among all the countries of the world in Human Development Index (UNDP). Moreover, Pakistan is considered among the low-income countries (LMIC) where more than $29.5 \%$ of people live below the poverty line which is defined as an income of less than $\$ 2$ per day.2 The health system of Pakistan is primarily governed by its provincial authorities though the government-led established public health care system in Pakistan in the 1960s and 1970s. 3However; health policy, decision-making, financing, budgeting, resource allocation, education and organizing public health initiatives, all such activities are predominantly governed at the federal level which is also responsible for regulation and coordination of health laws.

The main health system of Pakistan is divided into public sector and private sector. The public sector provides outreach health facilities at the primary, secondary and tertiary level. Initially, the primary health care services are provided through rural health centers (RHC) and basic health unit (BHU). District headquarters and tehsil headquarters provide secondary health care services and the rest of the population is catered by tertiary health care facilities which are affiliated with teaching hospitals in the urban cities. Some government organizations in Pakistan offer health services to their employees and their families which mainly include Pakistan military, airlines, and railway employees'. Furthermore, the functions of private health sector in Pakistan can be divided into formal and non-formal health sector. Overall, the health care expenditure share by the public sector is approximately $75 \%$ whereas; the private sector has $25 \%$ of its share in health care spending. Moreover small dispensaries, maternity care centers, specialized centers for heart diseases and kidney ailments, eye care centers were established by the government in addition. Traditional medicine has also been practiced in Pakistan which includes homeopathy, hikmat or eastern medicine that tends to use non-scientific methods and uses old traditional herbs and folk medicine ways to treat some diseases.

Similarly in the quest of achieving standards of quality health care like other countries of the developing world, Pakistan is struggling through its complex health care system to meet national goals as well as international commitments. Failing the target to achieve 'Health for All - 2000' resulted mainly due to lack of resources, lack of political will, lack of decisive actions and applicable policies. The aforementioned circumstances also play their enormous role in failing to attain the Millennium Development Goals (MDGs) by 2015. Importantly, failing MDGs was because of the uncertainty that was present among the political and economic spheres that impervious the health care situation in the country. A huge ethical dilemma captivating health system is the un sustained financing of health care programs. Health care financing may be referred as monetary funding that is utilized to effectively route a health-related initiative or an activity. However, major challenges to financing in Pakistan are predominantly related to prioritizing and allocating of required funds for the health programs. Being a low-income country (LIC), Pakistan faces inordinate number of challenges that mainly result from improper financing, budgeting and resource allocation for public health initiatives across the country.

Low health care spending by the government of Pakistan which is currently less than $1 \%$ with a minimal budget that is meagerly allotted to top priority prevention programs. In addition, $80 \%$ of the health care budget is spent on curative programs that are those provided at the secondary and tertiary level of care. Delivering quality health care services is often compromised and becomes ethically challenging because of limited resources, lack of accessibility and corruption that inhibits the health system from functioning effectively. Public health system not only is affected by these aforementioned factors but issues such as of health workforce, advancement of medical products and health care technologies, health care delivery, role of health information system and under representation of all stake holders in the decision making process cannot be repudiated in this context.4,5For an efficient public health system to work in any country, there lies a competitive demand for sincere public health workers, an honest representation by the government officials, a truthful say of community and all stakeholders and also the commitment from non-governmental organizations (NGOs).

It is often acclaimed that in order to improve the health status of any population which is directly proportional to the economic state of the country, a dedicated, honest and right commitment is needed by the government, principally. Then a question that is yet to be answered in the context of health system in Pakistan which gener- 
ally lies within the functioning of health care system is 'whether the government is really honest in providing and proclaiming equitable distribution of resources and service delivery while putting all the efforts? It is has been widely argued and anticipated that health care reforms occur at various levels of health care system which may be related to the administration or the organization's level or both. Ethically, health care reforms do not resist the citizens to attain their right to equitable health care in fact, the right to health is a basic human right which should not emerge as a commodity. Main goal of any public health initiatives are largely focused on prevention and on improving the health of the population. To inculcate lifestyles that are healthy for people, health guidelines, education and awareness are provided to the people which are intended to maximize the good for people. These educational guidelines allows people to understand and exercise their freedom in choosing what is good for them in order to maintain and restore their health on an individual level. Apart from diseasespecific prevention guidelines, strategies related to stress management, ensuring and encouraging healthy behaviors among people necessitates that they are made aware of such healthy activities.

In this regard, achievable success to any such health activity involves the participation of doctors and the community itself. Involving the community in the great good of prevention allows every member of the population to voluntary avail and reach such benefits. This can be further exemplified by secondary prevention measures that are aimed at early screening and diagnosing. By decreasing the extent of disability arising from a foreseen disease, it would be an ethical and moral obligation on the health authorities to launch and initiate screening programs in the population who may be apparently normal. This elucidates that following the moral duty of screening a healthy population for an anticipated disease outcome as been proclaimed in medical ethics, the right to administer treatment in times seem as desirable as well as preventing further progression of disease or disability in the subsequent population. Concerning the secondary level of prevention, involvement of physicians and nurses seem as necessary to halt the disease progression.

In Pakistan, the public health system is mainly consumed at the tertiary level where demarcation between the health services provision diminishes and borders between curative health care and prevention. An assessment of the needs of routine and uncomplicated patients is included in the primary care package which includes providing medical advice, diagnosis, treatment and referral care. These services are provided by the physicians and practioners who work in health care facilities and serve the catchment community or the population. Concerning unavailability of advanced medical technology, health care setup or medicines at the initial tier of the health system bounds the health care providers for referrals and for prompt treatment that is available at the immediate location. Equitable distribution of money, man power and resources proposes that at the primary care level such health services should be made available, accessible and in reach of the population. This can be further explained by the system of referral of patients from basic health units (BHU) to tertiary teaching hospitals in urban cities that involves complex procedures and medical services which a basic health unit (BHU) or a rural health center (RHC) may lack. In such a complex situation, availability of ambulatory services at the basic health units (BHU) particularly in emergency situations; jeopardizes the whole health care system which is meant to serve the public when they are in dire need of their basic health right. Consequently, the health care system of Pakistan can be placed in the category of 'poor permissive' where physical infrastructure is not sufficient to meet what people really need and require.

Regarding the public health system that is operational in Pakistan that has been able to launch many significant public health initiatives and campaigns which are counted among some of the successes by the health system. Such noteworthy initiatives cannot be marked as successful until and unless the role of donor agencies is commended and acknowledged. In order to meet the guidelines of World Health Organization (WHO) to achieve the international commitments, health system of Pakistan has been struggling long enough. Public health programs such as Expanded Program of Immunization (EPI), Malaria and Tuberculosis Control Programs, Family Planning and similar more initiatives have helped the country to reduce the burden of disease and to eliminate the prevalent infectious diseases in the masses. To mention some of the ethical challenges that may have compromised the progress of these programs are related to the deficits that are present in the health care system of Pakistan. This may include lack of physical infrastructure to function effectively especially where the distance to health facilities is far enough and it becomes difficult for the patients to travel and seek health care. Another soaring issue related to it is the mere presence of doctors and paramedical staff at these health facilities which at times were not functioning and ghost centers were in place in the documents. Moreover, the allocated funds were utilized with no intention to be efficiently spent for the health of poor. However, the state-owned health system has always considered 'Health for All' as an official policy working under every subsequent government. Apart from reality; despite of these issues of the health system, the public sector has been serving $85 \%$ of the population which mostly comprises of the poor population of Pakistan.

Due to these circumstances and obstacles in the health care delivery, the last two decades have witnessed the failure to public health programs and initiatives. This appended the private health sector to take on the opportunity and sell health as a business and for profit. With slight and hidden encouragement by the government, the private health sector have become the most desirable among the buyers of health in the country. Eventually, private sector has became one of the profitable businesses along with the encour- 
agement and help of dysfunctional and inefficient public health system which provided it with a ground to play and evolve. For the rest of the $85 \%$ of population, the private sector turned out to be expensive and became a significant barrier to access health care. The uncertainty of achieving public health goals and its failure are reflected through the health indicators which clearly showed significant health disparities and inequitable distribution of resources in the country. The dearth of health workforce according to the available data depicts that on average every 1500 persons have access to one doctor by patient-doctor ratio and one nurse for every 3700 patients, which hugely influences the functioning of health system and compromises efficient service delivery to people.

Among all the three tiers of health care system of Pakistan, numerous challenges have been identified whether related to human resource, allocation of available funds, scarcity of physicians in the market or lack of necessary infrastructure and shortage of essential medicines in public sector hospitals. Health care reforms and amendments in health laws have been claimed to complex the health system functioning, in addition. It has been argued that responsiveness should be addressed in view of constitutional amendments that have voiced the health sector reforms in Pakistan. It is also important to understand the complexity associated with the health reforms and the aspect of fairness in the health system and health care delivery. The main components that should be vigorously studied in view of ethical perspective includes fairness, adequacy and responsiveness that hugely determines the effective and efficient health care delivery in the country. In addition to this, an attempt to understand the public health system is however crucial in determining the position of health system to be in line with the national agenda and health priorities. It is though desirable and recommended that after the decentralization of health system in Pakistan, a coherence should be developed between the provinces so that they systematically focus towards solving the issues of managing the components of health care and capacity building within the health system. Concerning system related issues specifically, the lack of accountability in the health system is never truly addressed. Access to health is regressed by the unavailability of the services and the unfairness in the health care delivery. Health care reforms have essentially acclaimed to provide affordable, accessible and effective health care services to the population. However; this becomes difficult in the terrain of a complex and dysfunctional health system where accountability and transparency is ruined by treating people differently. With regard to the performance of the health system, equity with respect to the available resources becomes challenging and hence this is estimated as unfairness. Indicators that reflect the health of the population or health outcomes that indicate the utilization of health services in the country do play an immensely important role in determining the health seeking pattern of the population. This may also be seen as an imperial way of looking at the knowledge and awareness level of the people about the diseases. Since, an adequate level of utilization of health services may reflect that the people in the community are aware about the disease and they positively revert to seek treatment or at least get diagnosed for the disease in question.

Often social and cultural barriers are neglected that prevent the access to health among fractions of population. Public health system advocates that inter-sectoral collaboration should be exercised while planning for public health initiatives so that those section of population who are vulnerable and marginalized may access to health and can refute to financial obstacles and non-financial barriers that limit them to avail what they need to make their life and health better. Inter-sectoral approach is therefore used and employed for prevention programs that also effectively address the social determinants of life and health. Moreover, responsiveness is that only crucial element in delivering quality health care services that may be considered as a guaranteed component of health system functioning. In Pakistan, health care reforms were greatly needed and desired in order to sustain the improvement in the health of the population and the health care facilities. The health indicators were also among the consideration while supporting and advocating for health care reforms. Since, Pakistan is among the developing countries of the world and a low-income part of world's economy, the reality of health care reforms was mainly to increase the utilization of health services. But as the unemployment rate is quiet high in Pakistan which is a substantial part of social determinants that affect health, hence maximizing the outcome of every single penny that is afforded by the government or is spent out of the pocket by the afforded should be efficiently consumed. In this regard, government is seen as the guarantor for health and as the major buyer that controls the cost of health services and quality of care. The government aim is to ensure that the resources are maximized in organizing curative as well as preventive programs in the country. Not only this but it also aims to effectively address and function with the system to govern and provide affordable and quality health care which are offered with controlled management.

The struggling economy of Pakistan needed health care reforms when the health indicators of the country were on a greatly slow trail. Decentralization of health ministry and devolving financial and administrative powers to the provinces were the descendant to failing Millennium Development Goals (MDGs) 2015 particularly indicators of maternal and child health. The purpose that served devolution of Federal Ministry of Health was to abridge the health care system especially the structure of the management. This entirely was aimed to ensure effective and efficient health care delivery so as to improve the maternal and child health indicators in particular and to attain MDGs as well. Regarding the utilization of health system among the rural population, the devolution of all powers to the provinces was expected to be promising. Though, it needed deployment of health care workforce in the rural centers, provision of maternal and child health services, channelizing health financing in view of pro-poor and various related strategies 
to improve and upgrade the health information system in Pakistan. This in return imposed an obligation on the provincial authorities to adhere to the principles of fairness and equity in provision of health services and preventing the vulnerable i.e. mothers and their children from catastrophic health outcomes in the rural areas of all provinces.

Hence, rational decision-making in post-decentralization scenario is anticipated with the participation of all stake holders in improving the health indicators of the country so as to serve and attain national health priorities. Although, the distribution of power mainly was contained within the provinces and by the provinces which allowed them to control the health governance better than before. This also subjected them to face challenges and constraints in addressing the capacity issues of the health system and its functioning at the provincial level. The adoption of evidence-based approaches to identify key challenges related to equity and fairness in the health care system led to some potential gains by health planning at the district and provincial levels. Prioritizing specific programs such as related to maternal and child health led to implementation of effective health policies supplemented with program specific health information and material resources. Comparatively, the success of such public health initiatives can be determined by estimating the improvement in the health status of the population and by ensuring fairness in accessing health by the people. Decentralization of health system also provided us with a way forward to improve and upgrade the functioning of public health system by developing relevant policies and implementing them with true participation of all the stakeholders along with a determined political will in the country.

Volume 3 Issue 11 November 2019

(C) All rights are reserved by Subhana Akber. 